\title{
Measurement of Beacon Anisoplanatism Through a Two- Dimensional Weakly-Compressible Shear Layer
}

\author{
R. Mark Rennie ${ }^{1}$ \\ Center for Flow Physics and Control \\ University of Notre Dame, Notre Dame, IN, 46556 \\ Matthew R. Whiteley ${ }^{2}$ \\ MZA Associates Corporation, Dayton, OH, 45430 \\ ${\text { Garnett } \text { Cross }^{3} \text {, Dave Cavalieri }{ }^{4} \text {, Eric J. Jumper }}^{5}$ \\ Center for Flow Physics and Control \\ University of Notre Dame, Notre Dame, IN, 46556
}

An experimental investigation was conducted into the effectiveness with which aero-optic aberrations imposed on a collimated reference beam could be evaluated using a point-source beacon. The experiments were conducted in the University of Notre Dame's Compressible Shear-Layer Wind Tunnel which was used to create an optically-active shear-layer flow with high-speed Mach number of 0.8 . Anisoplanatic effects included a difference in wavefront shape between the (spherical wavefront) beacon and the (planar wavefront) reference beam, and a difference in the regions of the flow sampled by the beacon and reference beams. A modal compensation approach was used to minimize the anisoplanatism between the beacon and reference wavefronts, which showed that the best compensation results were obtained when the shear layer was regularized using mechanical forcing.

\section{Nomenclature}

$\begin{array}{ll}\boldsymbol{A} & =\text { modal estimation matrix } \\ \boldsymbol{c}_{h} & =\text { reference beam modal decomposition vector } \\ \boldsymbol{c}_{h} & =\text { estimated reference beam modal decomposition } \\ \boldsymbol{c}_{m} & =\text { measurement modal decomposition } \\ \varepsilon_{b}{ }^{2} & =\text { aperture-averaged phase variance for beacon } \\ \varepsilon_{h}{ }^{2} & =\text { aperture-averaged phase variance for reference beam } \\ \varepsilon_{h b}{ }^{2} & =\text { anisoplanatic variance } \\ f & =\text { focal length } \\ f_{f} & =\text { shear-layer forcing frequency } \\ \phi_{b} & =\text { phase error imparted to beacon } \\ \phi_{h} & =\text { phase error imparted to reference beam } \\ L & =\text { distance from aperture to target } \\ L_{a} & =\text { length scale from aperture to aero-optic disturbances } \\ L_{b} & =\text { distance from aperture to beacon } \\ \Lambda & =\text { shear-layer structure wavelength }\end{array}$

\footnotetext{
${ }^{1}$ Assistant Research Professor, Department of Aerospace and Mechanical Engineering, Senior Member AIAA.

${ }^{2}$ Vice-President, Senior Scientist, MZA Dayton Operations, Member AIAA.

${ }^{3}$ Graduate Research Assistant, Department of Aerospace and Mechanical Engineering, Student Member AIAA.

${ }^{4}$ Research Specialist, Department of Aerospace and Mechanical Engineering, Senior Member AIAA.

${ }^{5}$ Professor, Department of Aerospace and Mechanical Engineering, Fellow AIAA.
} 


$\begin{array}{ll}M_{1} & =\text { Mach number of low-speed side of shear layer } \\ M_{2} & =\text { Mach number of high-speed side of shear layer } \\ O P D & =\text { optical path difference } \\ x & =\text { streamwise distance from splitter trailing edge } \\ y & =\text { cross-stream dimension }\end{array}$

\section{Introduction}

High field-of-regard, aircraft-mounted laser systems typically include parts of the operating envelope in which the laser must pass through highly-turbulent flow regions, such as a turbulent boundary layer or a shear layer associated with a separated flow region [1]. At subsonic and higher flight speeds, these turbulent flow regions become optically active such that the transiting laser beam will be distorted due to index-of-refraction variations within the flow $[2,3]$. The study of the optical aberrations produced by these kinds of nearfield turbulent flows is called "aero-optics."

The phase characteristics of the initial light beam can be restored using an adaptive-optic (AO) system [4] that places the conjugate waveform of the aberration onto the optical wavefront of the beam prior to its transmission through the aberrating flowfield. In this case, computation of the conjugate waveform is predicated on an accurate determination of the original aero-optic aberration. Even for feed-forward AO correction schemes, in which flowcontrol techniques are used to improve the frequency bandwidth of the aberrating flow, it is still anticipated that low-order optical measurements will still be used to synchronize the AO scheme with the controlled flow aberrations [5].

One method of measuring the aero-optic aberrations is to use the light from a guide star [6]. The guide star may be either a naturally-occurring nearby star or generated artificially using either light beacons mounted on the aircraft itself or a high-power laser beam that is focused to induce free-space air breakdown. For all types of guide stars, anisoplanatism effects occur when the light from the guide star does not pass through the same aero-optic flow as the outgoing laser beam. Further, laser-generated guide stars (LGS) have additional anisoplanatism effects because of the close proximity of the LGS which results in noticeable wavefront curvature. In this paper, experimental data are presented of anisoplanatism effects that were investigated for a simulated LGS that was used to interrogate an optically-active high-subsonic compressible shear layer.

\section{Approach}

\section{A. Anisoplanatism Effects}

\section{Focal Anisoplanatism with Aero-Optics}

The analysis geometry for beam propagation through an aero-optic disturbance is shown in Fig. 1. The figure shows an aero-optics beacon located at a short distance compared to the propagation range of the outgoing laser, which is used to correct the aero-optic aberrations of the outgoing laser. The outgoing beam is imparted with a phase error $\phi_{h}$ due to aero-optic disturbances in the propagation path, while the beacon reference wave is imprinted with a phase error $\phi_{b}$; the aperture-averaged phase variances for the outgoing beam and beacon are therefore:

$$
\begin{aligned}
& \varepsilon_{h}^{2}=\int d \rho W(\rho)\left\langle\left[\phi_{h}(R \rho)\right]^{2}\right\rangle \\
& \varepsilon_{b}^{2}=\int d \rho W(\rho)\left\langle\left[\phi_{b}(R \rho)\right]^{2}\right\rangle
\end{aligned}
$$

where $W(\rho)$ is an aperture-averaging mask. As the primary error metric for AO phase compensation, the "anisoplanatic variance" can be defined as:

$$
\varepsilon_{h b}^{2} \equiv \int d \rho W(\rho)\left\langle\left[\phi_{h}(R \rho)-\phi_{b}(R \rho)\right]^{2}\right\rangle
$$


This error metric can be further broken down into distinct components:

$$
\varepsilon_{h b}^{2}=\tilde{\varepsilon}_{h b}^{2}+\bar{\varepsilon}_{h b}^{2}
$$

where the term $\tilde{\varepsilon}_{h b}{ }^{2}$ represents the variance of aero-optics phase not measured because of the finite range $L_{b}$ of the beacon, and the term $\bar{\varepsilon}_{b}^{2}$ represents the variance of phase measured in error by the beacon due to probing a different aero-optics disturbance volume than the outgoing beam focused on a target at range $L$. Relating each of the components of residual error to characteristics of the propagation path depends on the distribution of the aero-optics disturbances over the path characterized by the scale length $L_{a}$.

A key quantity in the analysis of aero-optics anisoplanatism is the fractional differential phase variance between the beacon and collimated reference beam at each point within the beacon reference volume; that is, the term $\left\langle\left[\phi_{h}(R \rho)-\phi_{b}(R \rho)\right]^{2}\right\rangle$ in Eq.(3). This is an intrinsic property of the aero-optical flow for which no theoretical expressions exist. However, insight into the nature of this quantity was obtained from an analysis of laboratory wind tunnel data collected by the University of Notre Dame [1] and employed in other aero-optics efforts

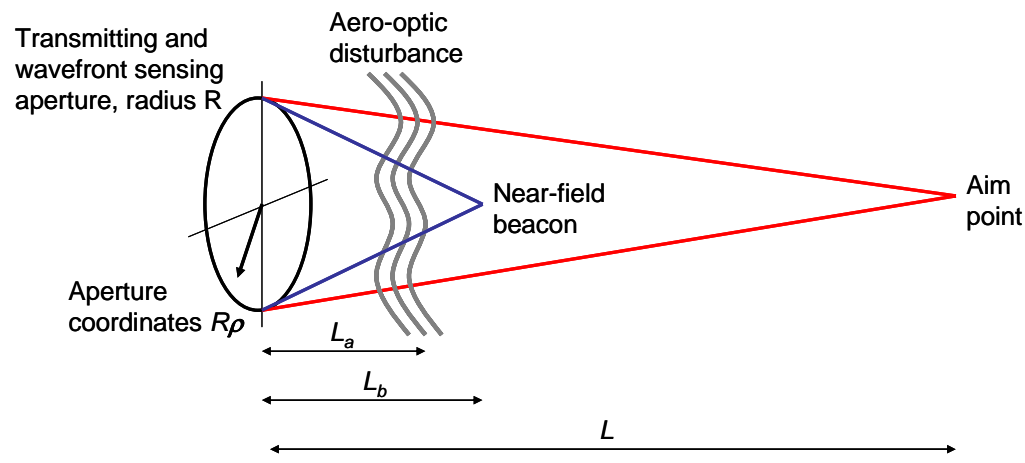

Figure 1. Analysis geometry for correction of aero-optic aberrations using near-field beacon.
[7]. These data showed that for a near-field beacon (i.e. $L_{b} / L<0.1$ ) and with $L_{b} / L_{a} \leq 2$, anisoplanatic compensation is no better, or marginally worse, than open-loop propagation. Hence, AO compensation using a beacon at this range would result in essentially no reduction of aero-optical effects. For the total focal anisoplanatism to be less than half the open-loop disturbance level requires $L_{b} / L_{a}>3$, and to essentially eliminate degradation due to focal anisoplanatism requires $L_{b} / L_{a}>10$.

\section{Mitigation of Focal Anisoplanatism}

Increasing the range of the beacon relative to the aero-optics disturbance location $L_{a}$ may appear to be an easy solution to the problem of measurement anisoplanatism, but will have practical limits. An alternate approach to mitigating the anisoplanatic degradation associated with a beacon located at $L_{b} / L_{a} \sim 2$ is the use of optimal estimation. Optimal modal compensation $[8,9]$ is a method that offers considerable advantage and flexibility to the problem of aero-optics focal anisoplanatism. In summary, this technique uses a linear estimation matrix $\boldsymbol{A}$ applied to a modal measurement set $\boldsymbol{c}_{m}$ consisting of $N$ modes from one or more reference beacons to yield an estimate for the desired correction:

$$
\hat{\boldsymbol{c}_{h}}=\boldsymbol{A} \boldsymbol{c}_{m}
$$

By the nature of the optimality criterion for the estimator, the residual modal anisoplanatism is indeed minimized, given the properties of the measurements and their relation with the desired phase correction. The minimum error resulting from application of the estimator is given by

$$
\operatorname{diag}\left[\left\langle\left(\boldsymbol{c}_{h}-\hat{\boldsymbol{c}}_{h}\right)\left(\boldsymbol{c}_{h}-\hat{\boldsymbol{c}}_{h}\right)^{T}\right\rangle\right]=\operatorname{diag}\left[\left\langle\boldsymbol{c}_{h} \boldsymbol{c}_{h}^{T}\right\rangle-\boldsymbol{B} \boldsymbol{C}^{-1} \boldsymbol{B}^{T}\right]
$$

This minimization requirement corresponds exactly with the optimality criterion of a minimal mean-square error (MMSE) estimator for the reference beam modes. Forming such an estimator requires calculation of the covariance 
of the beacon measurement modes as well as the cross-covariance of the measurements with the desired correction, as follows:

$$
\begin{aligned}
& \boldsymbol{A}=\boldsymbol{B} \boldsymbol{C}^{-1} \\
& \boldsymbol{B}=\left\langle\boldsymbol{c}_{h} \boldsymbol{c}_{m}{ }^{T}\right\rangle \\
& \boldsymbol{C}=\left\langle\boldsymbol{c}_{m} \boldsymbol{c}_{m}{ }^{T}\right\rangle
\end{aligned}
$$

These statistical quantities depend primarily upon the location of the aero-optics beacon relative to the characteristic location of the aero-optics disturbance, $L_{b} / L_{a}$, and secondarily to the location of the beacon relative to the overall path length, $L_{b} / L$.

\section{B. Experiment}

The beacon anisoplanatism measurements were performed in the University of Notre Dame's Compressible Shear-Layer Wind Tunnel (CSLWT), which was constructed specifically to investigate the optical characteristics of high-subsonic compressible shear layers. The CSLWT mixes co-directional high- and low-speed flows at high subsonic flow speeds (up to Mach 1.0) to create a shear layer that is aero-optically active, and representative of the kinds of aero-optic flows likely to be encountered on flight vehicles. Details on the design and flow characteristics of the CSLWT can be found in $[5,10]$.

Anisoplanatism effects were evaluated by comparing wavefront aberrations from a point-source "beacon" to those from a collimated reference laser beam after passing through the compressible shear-layer flow. Wavefronts were measured using a Shack-Hartmann-type wavefront sensor manufactured by Wavefront Sciences. The flow was interrogated using a $532 \mathrm{~nm}$ wavelength frequency-doubled pulsed Nd:YAG laser. The wavefront sensor was a CCD camera with a 33 x 44 lenslet array and, although the frame rate of the CCD camera was only $30 \mathrm{~Hz}$, the $M_{2}=0.78$ flow was effectively frozen by the 8 ns laser pulses.

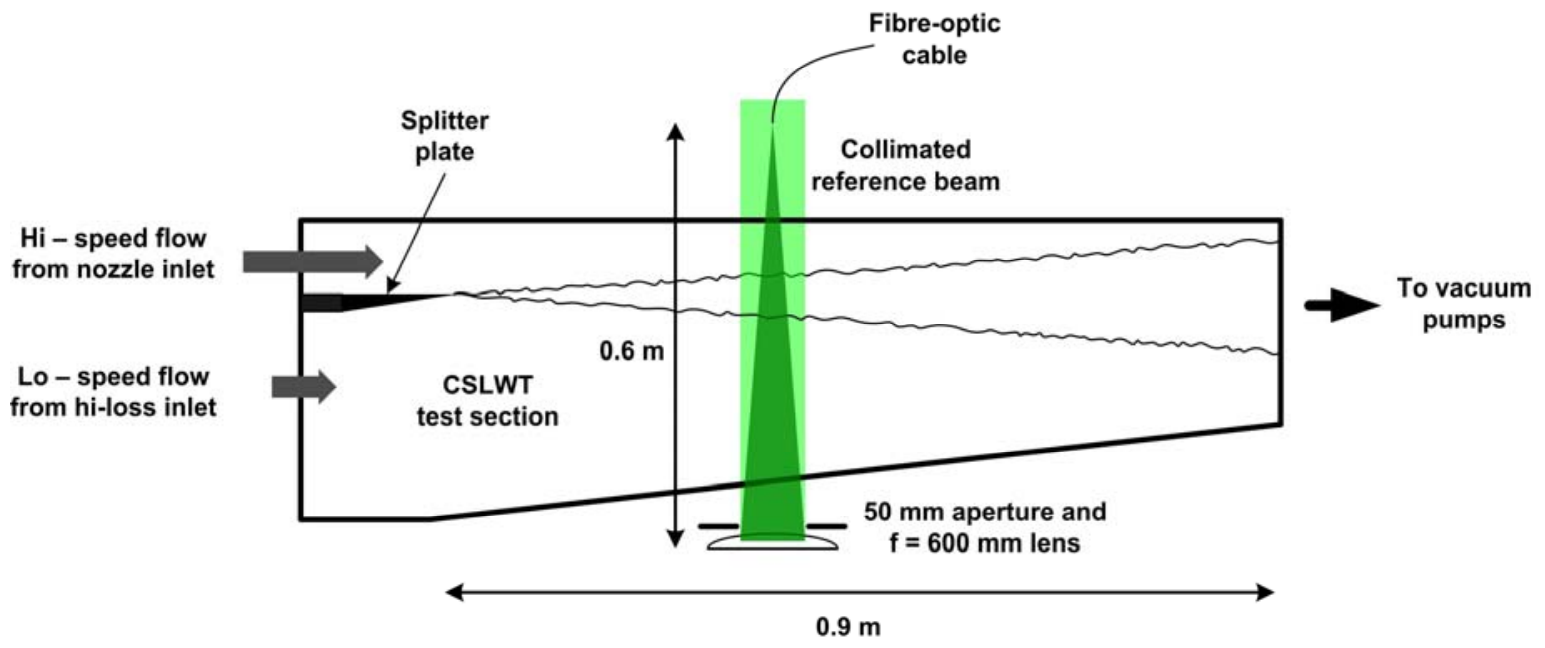

Figure 2. Basic geometry of beacon anisoplanatism measurement

In practice, laser guide stars are generated by projecting a focused laser beam into the atmosphere, typically within the field of view of the correcting AO system. The focused laser beam is of sufficient intensity to induce air breakdown, creating a nearly point source of light which is then used to measure the aero-optic environment in the vicinity of the outgoing laser beam aperture. For our tests, the LGS was simulated using the light projected from an optical fiber that was aimed along the axis of the optical setup. The fiber used in the tests was a single mode fiber with core diameter of $3.6 \mu \mathrm{m}$. The light emitted from a cleaved end of the fiber diverged with a full angle of approximately $5^{\circ}$.

An overview of the basic geometry of the experimental setup is shown in Fig. 2. A 50 mm diameter collimated reference beam was passed through the shear layer co-axially with the diverging beacon beam emanating from the fiber. A $f=600 \mathrm{~mm}$ lens, apertured to $50 \mathrm{~mm}$, was located a distance $600 \mathrm{~mm}$ away from the fiber in order to 
collimate the beacon beam after passing through the shear layer. The shear layer, which contributed most of the aero-optic aberrations to the reference and beacon beams, was situated halfway between the fiber and the $f=600 \mathrm{~mm}$ lens; as such, due to the geometry of the setup, the diameter of the beacon beam as it passed through the aberrating shear layer was approximately half that of the reference beam. This geometrical difference in the beacon and reference beams, in addition to the difference in curvature of the beacon (spherical wavefronts) and reference (planar wavefronts) beams, constituted the anisoplanatic effects that were evaluated by the measurements.

A photograph of the optical setup is shown in Fig. 3. The setup consisted of transmission optics mounted on a raised platform that was level with the top of the CSLWT test section, and receiving optics mounted below the test section. The transmission optics included the laser, a beam splitter, a fiber-optic coupler and single-mode fiber to generate the simulated diverging beacon signal, and a series of beam expanders that generated the $50 \mathrm{~mm}$ collimated reference beam. The beacon and collimated beams were directed down through the test section by a mirror.
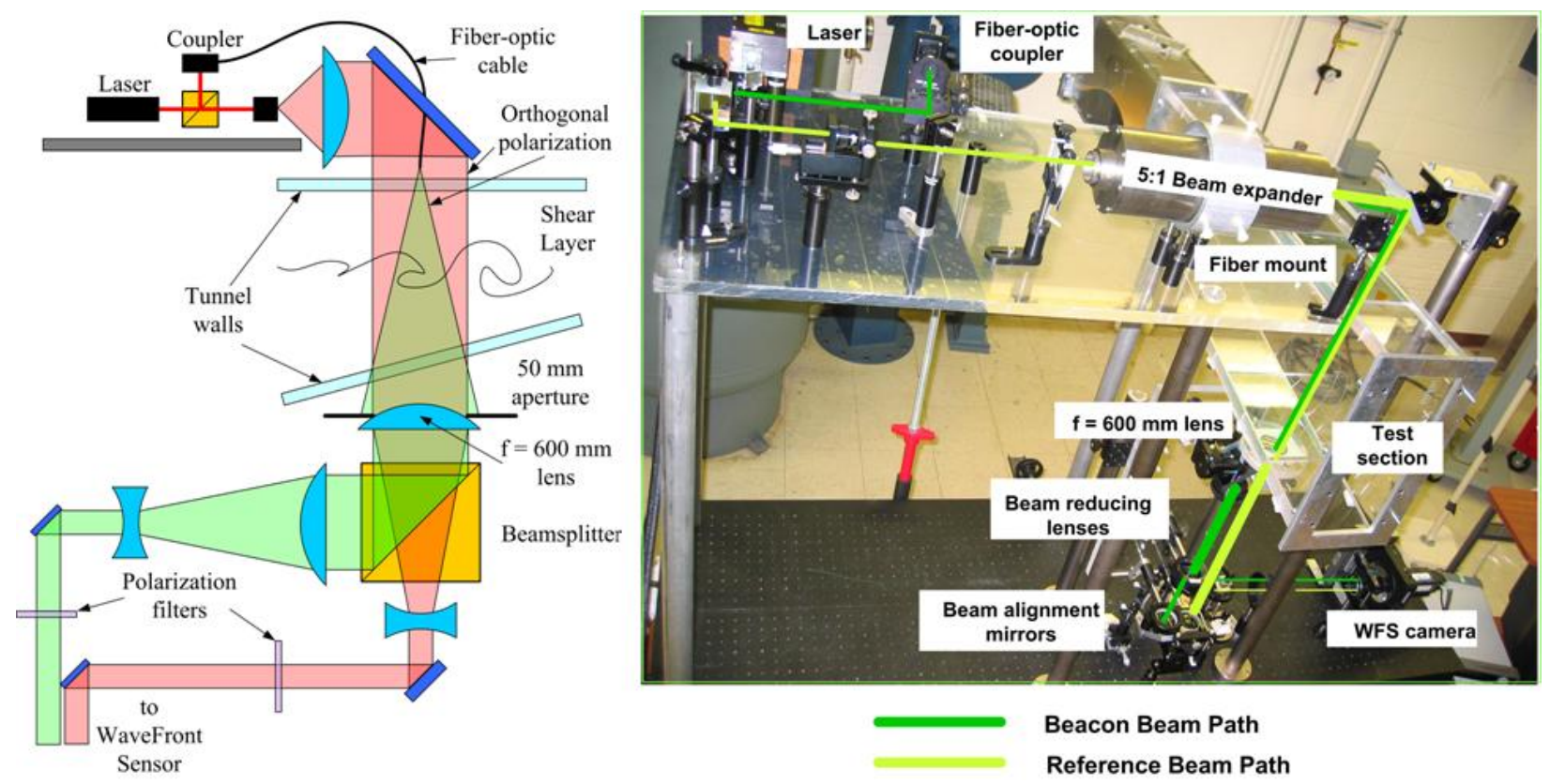

Figure 3: Schematic (left) and photograph (right) of optical setup

The receiving optics underneath the test section were designed to separate, reduce, and align the beacon and reference beams into the wavefront sensor. The first element of the receiving optics was the $600 \mathrm{~mm}$ focal length lens apertured to $50 \mathrm{~mm}$. This lens both collimated the beacon beam and acted as the first element of a 5:1 beam reducer for the reference beam. Just after the $600 \mathrm{~mm}$ lens, a beam splitter was used to direct the collected light into two different lens systems that reduced the apertured beacon and reference beams into (approximately) 0.5-in diameter beams. At this stage, both the beacon and reference beams were passed into both beam-reducer lens systems; however, the different starting wavefront shape of each beam (i.e. the collimated planar reference wavefront versus the diverging spherical beacon wavefront) effectively filtered the incorrect beam out of each beam reducer, leaving no noticeable cross talk in the wavefront sensor images. The two 0.5 -in beams were then guided using mirrors so that the two beams were projected side by side into the wavefront sensor. To enable alignment of the two beams and thus minimize differences in no-flow tip/tilt between the two beams, one of the mirrors was mounted on a translation stage. The final optical element was a 4:1 beam reducer that projected the two aligned 0.5 -in beams into the WFS camera. Test images acquired with the WFS camera with the flow off showed no noticeable spherical aberrations, and verified that the beacon image covered approximately the inner $50 \%$ of the reference beam at the shear layer location. 


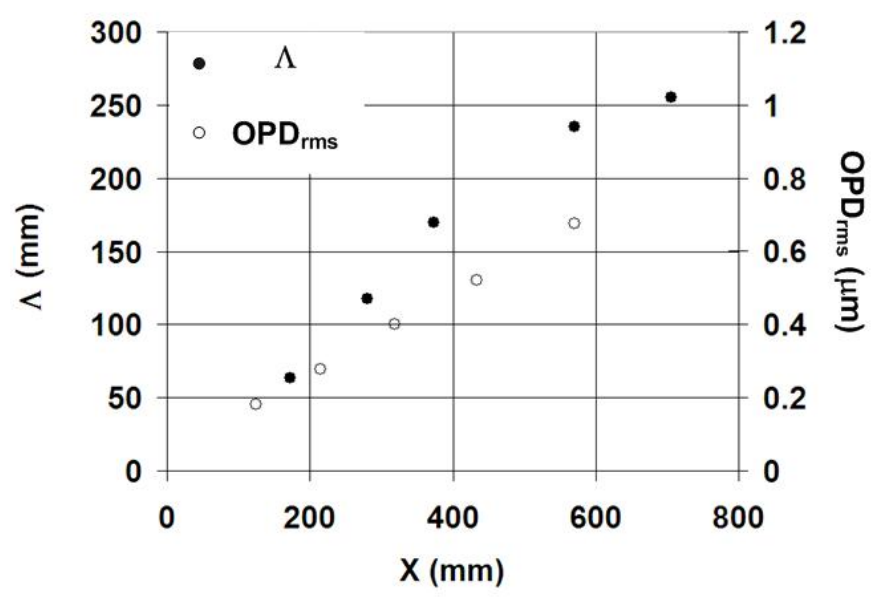

Figure 4: Dominant shear-layer structure size (unforced) and OPDrms in CSLWT, $M_{2}=0.78 / M_{1}=0.15$

\section{Measurements}

In order to generate strong aberrations in the shear-layer flow and hence good signalto-noise ratio, the wind tunnel was run at close to its maximum wind speed with $M_{2}=0.78, M_{1}=0.15$. This operating point gives a shear layer velocity ratio $r \approx 0.15$, which is representative of the kinds of shear layers that are created by separated flow regions, and has been used in other investigations [5, 11].

As shown in [3], optical aberrations in the shear layer are caused primarily by the lowpressure wells in the center of vortical structures within the shear layer. The dominant vortical structure size (and concomitant aberration size) in the shear layer scales with the shear-layer cross-stream width, which increases linearly in the streamwise direction. To obtain an indication of aberration size on the measured anisoplanatism, data were acquired at several downstream locations.

The dominant shear-layer structures size in the CSLWT at the test speed $M_{2}=0.78 / M_{1}=0.15$ is plotted in Fig. 4; which also shows the OPDrms of the related aberrations in order to give an indication of the strength of the optical signal. The figure shows that, at small streamwise distances, the structures sizes are very small and result in smallamplitude optical aberrations, while farther downstream the structures become larger and produce strong aberrations. At the most upstream locations, the optical aberrations were too weak to be accurately measured by the WFS, so that measurements were made downstream of $x=200 \mathrm{~mm}$. On the other hand, at the farthest downstream locations, the $50 \mathrm{~mm}$ aperture of the optical setup was too small to capture the entire aberration size of the much larger, downstream aberrations. The result was that at the farthest downstream locations, the $50 \mathrm{~mm}$ aperture effectively "filtered" the large-scale aberrations so that the aberrations appeared primarily as tip/tilt within the $50 \mathrm{~mm}$ aperture. Further, because the entire aberration did not appear within the aperture, the OPD measured by the WFS was also reduced. As such, to avoid excessive aperture effects, the maximum downstream location at which measurements were made was limited to $x=400 \mathrm{~mm}$.

Measurements were also performed with the shear layer forced at several different forcing frequencies. The shear layer was forced using voice-coil actuators that applied a moving-boundary perturbation to the flow perpendicular to the flow direction at the trailing edge of the splitter plate $[5,10]$. The shear layer was forced over the most-effective frequency range of the forcing actuator, $750 \mathrm{~Hz}$ to 1500 $\mathrm{Hz}$, producing regularized shear-layer structure (and aberration) sizes from 100 to $200 \mathrm{~mm}$. The shear-layer forcing also produced much stronger OPD's and improved signal-to-noise ratios for the wavefront measurements. The test matrix of anisoplanatism measurements that were performed is summarized in Table 1.

\begin{tabular}{|l|l|c|}
\hline$X(\mathbf{m m})$ & $\begin{array}{l}\text { Forcing } \\
(\mathbf{H z})\end{array}$ & $\begin{array}{l}\text { Dominant } \\
\text { Aberration } \\
\text { Size } \Lambda(\mathbf{m m})\end{array}$ \\
\hline 200 & None & $\mathbf{8 0}$ \\
\hline & 750 & 200 \\
\hline & 1000 & 160 \\
\hline & 1500 & 100 \\
\hline 300 & None & 120 \\
\hline & 750 & 200 \\
\hline & 1000 & 160 \\
\hline & 1500 & 100 \\
\hline 400 & None & 180 \\
\hline & 750 & 200 \\
\hline & 1000 & 160 \\
\hline & 1500 & 100 \\
\hline & &
\end{tabular}

Table 1: Test conditions for anisoplanatism measurements 


\section{Results}

An example of wavefronts from the beacon and reference beams is shown in Fig. 5. The wavefronts were acquired at a downstream location $x=300 \mathrm{~mm}$, and with the shear-layer forced at a frequency of $750 \mathrm{~Hz}$. The wavefront for the beacon beam in the figure has been scaled to a diameter of $25 \mathrm{~mm}$, which is the diameter of the beacon beam as it passes through the optically-active region of the shear-layer flow. As can be seen in the figure, the $50 \mathrm{~mm}$ aperture of the optical system captures only a small portion of the $200 \mathrm{~mm}$ long aberration wavelength associated with the $750 \mathrm{~Hz}$ forcing, with the result that the aberration appears mostly as a streamwise tilt. The figure shows that the aberration detected using the beacon closely matches the inner $50 \%$ of the wavefront measured by the reference beam.
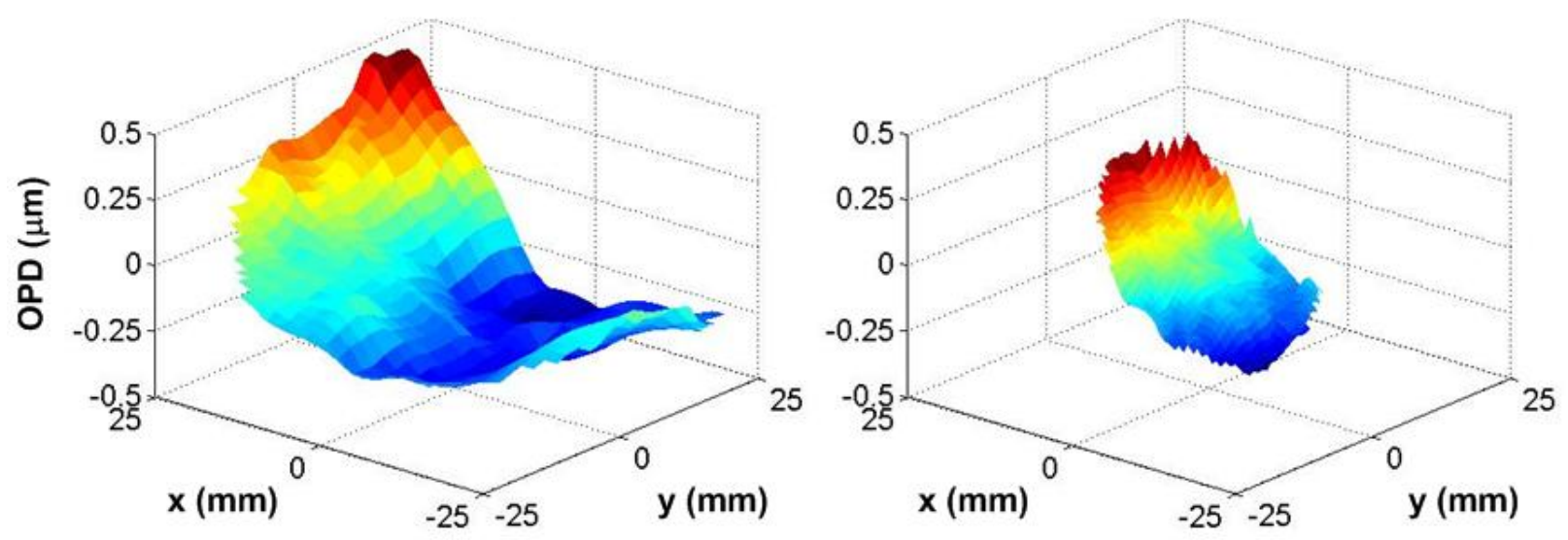

Figure 5: Sample wavefronts for collimated reference beam (left) and beacon beam (right) acquired at $x=300 \mathrm{~mm}$ and with shear layer forced at $750 \mathrm{~Hz}$.

\section{A. Mitigation of Anisoplanatism by Optimal Estimator}

\section{Unforced Shear Layer}

The results of the anisoplanatism measurements for the unforced shear layer are summarized in Fig. 6. The figure shows, first, the aperture-averaged phase variances measured for the reference and beacon beams (blue and green lines). The phase variances were computed for $1 \mu \mathrm{m}$ radiation, with tip/tilt removed, and are shown as the average of 50 individual wavefront measurements. As can be seen in the figure, the variance for the reference and beacon beams increases in the streamwise direction, which is an expected outcome of the increasing shear-layer structure size and concomitant aberration strength as the shear-layer evolves in the downstream direction (cf Fig. 4). Also plotted in Fig. 6 are the anisoplanatic variance $\varepsilon_{h b}{ }^{2}$ and the residual anisoplanatism after correction using the MMSE estimator, $\hat{\varepsilon}_{h b}{ }^{2}$ (red and black lines). The anisoplanatic residual after MMSE estimation $\hat{\varepsilon}_{h b}{ }^{2}$ was computed by estimating the reference wavefront mode vector $\hat{\boldsymbol{c}}_{h}$ via Eq. (5), constructing estimated phase error distributions for the reference beam, $\hat{\phi}_{h}$, and substituting these estimated phase error distributions into Eq. (10):

$$
\hat{\varepsilon}_{h b}^{2} \equiv \int d \rho W(\rho)\left\langle\left[\phi_{h}(R \rho)-\hat{\phi}_{h}(R \rho)\right]^{2}\right\rangle
$$

Figure 6 shows that, at the most upstream locations, the anisoplanatism of the uncompensated data is almost as large as the wavefront variance of the reference beam itself. This result shows that even though it is easily possible to discern the similarities between wavefronts for the beacon and reference beams for individual measurements as shown in Fig. 5, the differences between the two wavefronts that arise from the various sources of anisoplanatism (eg. $\tilde{\varepsilon}_{h b}{ }^{2}+\bar{\varepsilon}_{h b}{ }^{2}$ in Eq. (4)) are still significant. As such, use of the unmodified beacon wavefronts to estimate the 


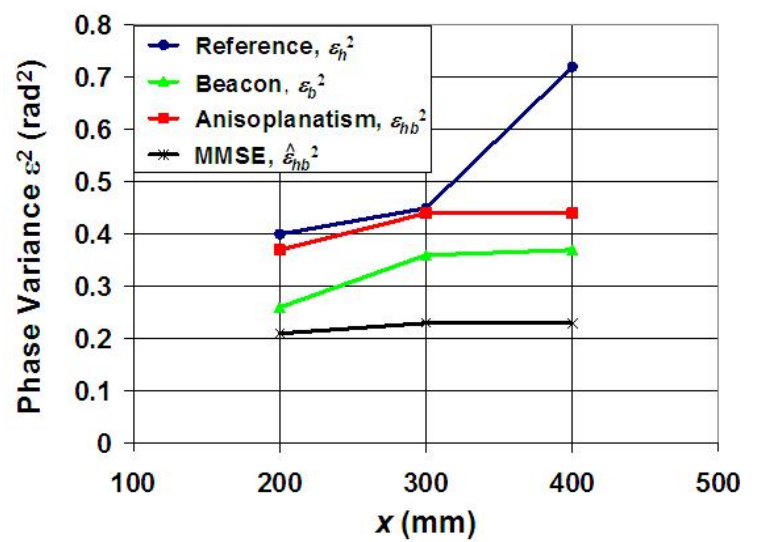

Figure 6: Anisoplanatism results for unforced shear layer.

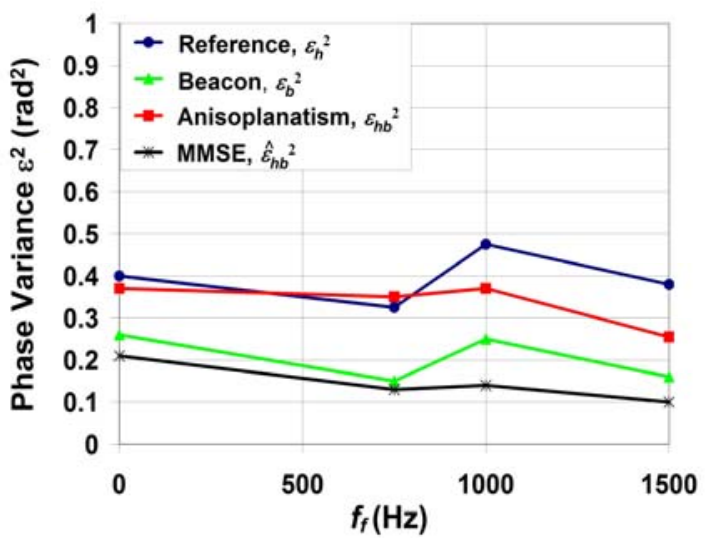

Figure 7: Anisoplanatism results for the forced shear layer, $x=200 \mathrm{~mm}$.

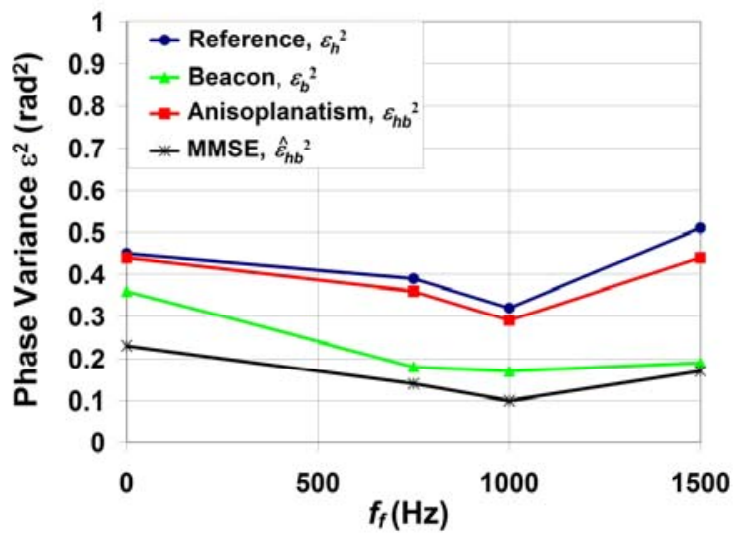

Figure 8: Anisoplanatism results for forced shear layer, $x=300 \mathrm{~mm}$. reference beam phase variance (eg. for the purpose of AO correction) would result in additional errors from the estimation that could be of the same order as the original reference beam variance. On the other hand, Fig. 6 shows that the MMSE estimator reduced the anisoplanatism by a factor of 2 or more, so that the residual anisoplanatism between the corrected beacon wavefronts and the reference wavefronts ranges from approximately $50 \%$ to less than $30 \%$ of the original reference beam wavefront variance.

\section{Forced shear layer}

Anisoplanatism results are shown in Figs. (7) to (9) for the shear layer forced at $750 \mathrm{~Hz}, 1000 \mathrm{~Hz}$ and $1500 \mathrm{~Hz}$, at the $x=200 \mathrm{~mm}, 300 \mathrm{~mm}$ and $400 \mathrm{~mm}$ measurement stations. The results also show a slight reduction in the residual anisoplanatism $\hat{\varepsilon}_{h b}{ }^{2}$ when the shear layer is forced; this trend can be more clearly observed by the slight upward slant of the data in Fig. 10 which plots the reduction in anisoplanatism realized by the MMSE estimator, $\varepsilon_{h b}{ }^{2}-\hat{\varepsilon}_{h b}{ }^{2}$. This reduction in residual anisoplanatism is likely due to the higher amplitude and improved signal to noise of the forced aberrations. More significantly, the improved performance of the MMSE estimator might also be an outcome of the regularized nature of the forced shear, in which random disturbances are suppressed in favor of the large-amplitude vortical structures with passing frequency dictated by the forcing frequency; the optical aberrations of these large vortical structures would show much greater correlation in the beacon and reference wavefronts once anisoplanatic effects are removed. 


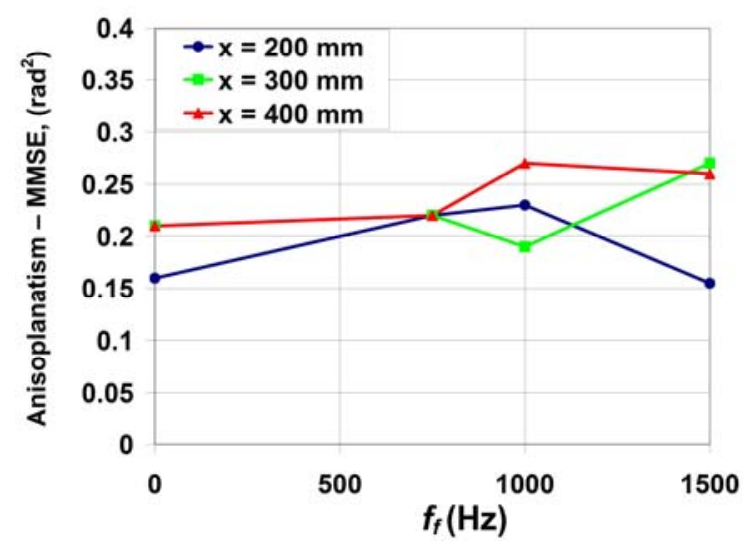

Figure 10: Reduction of anisoplanatism by MMSE estimator.

\section{Discussion}

The data presented in this paper have illustrated the success of the MMSE estimation technique in reducing anisoplanatism between the point-source beacon and collimated reference beams. In this regard, these data represent the first step towards developing near-field beacons for AO correction of HEL systems.

As detailed in the experiment description, due to the small size of the measurement aperture, the aberrations imposed on both the beacon and reference wavefronts by the shear layer were primarily streamwise tilt. As such, future studies should be directed towards increasing the ratio of the aperture size to the dominant shear-layer aberration scale, in order to more fully determine the effect of this parameter on the performance of the MMSE technique. The results of this investigation have shown, however, that regularization of the shear layer by forcing changes the statistics of the anisoplanatism so that the estimator reduces the anisoplanatism more effectively; this synergy between shearlayer forcing and the optimal estimation technique suggests that the two approaches might be used concurrently. In this case, it should be noted that the forced shear layer produces strong, coherent and sinusoidal-like aberrations [5], and simple studies indicate that the MMSE technique should work well for these kinds of aberrations at any scale.

As a final comment, it should be noted that correction of the beacon measurements using the MMSE technique relies on the availability of a simultaneously-measured set of beacon and reference wavefronts in order to evaluate the anisoplanatism of the beacon beam and construct the estimation matrix; this requirement is apparent from the definition of the $\boldsymbol{B}$ matrix, Eq. (8). For the results presented in this paper, separate sets of beacon and reference wavefronts were acquired for each test condition (streamwise location and forcing frequency), and a distinct estimation matrix $\boldsymbol{A}$ was computed and used to correct the beacon anisoplanatism. For a flight application, the optical configuration could be "calibrated" in a set of pre-flight experiments to generate a database of estimation matrices that cover a specified range of flight conditions. Future work on this topic might therefore investigate issues such as the minimum requirements for such a database, and the ability to interpolate or extrapolate from calibrated flight conditions to untested conditions.

\section{Acknowledgments}

These efforts were sponsored by the Air Force Research Laboratory Directed Energy Directorate under Contract Number FA9451-07-M-0078. The U.S. Government is authorized to reproduce and distribute reprints for governmental purposes notwithstanding any copyright notation thereon.

\section{References}

[1] Gordeyev, S., Post, M.L., McLaughlin, T., Ceniceros, J., and Jumper, E.J., "Survey of Optical Environment over Hemisphereon-Cylinder Turret Using Suite of Wavefront Sensors," AIAA-2006-3074, June, 2006.

[2] Gordeyev, S., and Jumper, E.J., "Aero-Optical Characteristics of Compressible Subsonic Turbulent Boundary Layers," AIAA-2003-3606, June, 2003.

[3] Fitzgerald, E.J. and Jumper E.J., "The optical distortion mechanism in a nearly incompressible free shear layer," Journal of Fluid Mechanics, Vol. 512, 2004, pp. 153-189.

[4] Tyson, R.K., Principles of Adaptive Optics, Academic Press, Inc., San Diego, 1991.

[5] Rennie, R. M., Duffin, D.A., and Jumper, E. J., "Characterization of a Forced Two-Dimensional, Weakly-Compressible Subsonic Free Shear Layer," AIAA 2007-4007, June, 2007.

[6] Whiteley, M.R., Welsh, B.M., and Roggemann, M.C., "Optimal modal wave-front compensation for anisoplanatism in adaptive optics,” J. Opt. Soc. Am. A, 15, no.8, Aug. 1998, pp 2097-2106. 
[7] Whiteley, M.R., Magee, E.P., Gibson, J.S., and Suizu, R.I., "Adaptive Controls for Aero-Optics and Atmospheric Compensation,” Technical Report AFRL-DE-PS-TR-2007-1044, Air Force Research Laboratory, Directed Energy Directorate, Kirtland AFB, New Mexico, 2007. Contract Number FA9451-06-M-0128, MZA Associates Corporation.

[8] Whiteley, M.R., Magee, E.P., Gibson, J.S., and Suizu, R.I., "Adaptive Controls for Aero-Optics and Atmospheric Compensation," Technical Report AFRL-DE-PS-TR-2007-1044, Air Force Research Laboratory, Directed Energy Directorate, Kirtland AFB, New Mexico, 2007. Contract Number FA9451-06-M-0128, MZA Associates Corporation.

[9] Kay, S.M., Fundamentals of Statistical Signal Processing: Estimation Theory, Prentice Hall, NJ, 1993.

[10] Rennie, R. M., Siegenthaler, J. P., and Jumper, E. J., "Forcing of a Two-Dimensional, Weakly-Compressible Subsonic Free Shear Layer," AIAA 2006-0561, Jan., 2006.

[11] Hugo, R.J, Jumper, E.J., Havener, G., and Stepanek, C., "Time-resolved Wave Front Measurements Through a Compressible Free Shear Layer,” AIAA Journal, Vol. 35, No. 4, 1997, pp 671-677. 